\title{
APRECIACIONES Y ALCANCES DE LA GESTIÓN DEL CONOCIMIENTO Y DEL CAPITAL INTELECTUAL EN LA EDUCACIÓN CONTINUA DEL CONTADOR PÚBLICO
}

Dr. Ernesto Augusto Polar Falcón*

\begin{abstract}
RESUMEN
El presente artículo, tiene por objetivo mostrar al Contador Público Colegiado los caminos de estudio que puede incursionar para el desarrollo de la gestión del conocimiento y del capital intelectual de una empresa; y mantener su nivel profesional debidamente actualizado en el marco de la educación continua profesional, como un deber ineludible que tiene un beneficio del crecimiento cualitativo profesional del Contador Público Colegiado y el ingreso del Perú en los procesos del área del conocimiento aplicados a las empresas nacionales que desean alcanzar un alto nivel de competitividad.
\end{abstract}

\section{INTRODUCCIÓN}

En el Anteproyecto del nuevo perfil de Contador Público que propone la Universidad de Puerto Rico $^{1}$, plantea el alcance de quince capacidades, todas ellas interesantes y de un gran contenido moderno e innovador. La primera capacidad está referida a alcanzar un "espiritu investigativo" y la segunda menciona que sea "un autogestionador de un proceso educativo continuo". Asimismo, también plantea incluir quince elementos en el perfil de la metodologia de enseñanza para el nuevo milenio, entre los cuales destacamos los siguientes:

- Aprendizaje participativo

- Pensamiento crítico

- Experiencias de investigación

- Evaluación crítica y analítica de literatura reciente

- Aprendizaje creativo

- Sed insaciable por el conocimiento

- Proyectos de servicio-aprendizaje

- Proyectos de aprendizaje por vivencia personal

- Profesor Principal de la Facultad de Ciencias Contables y Director del Instituto de Investigaciones de Ciencias Financieras y Contables

1 Currículos para un Profesional de Clase Mundial - Área y - Educación - Grupo 4.1 de Trabajos Nacionales XXIII-Conferencia Internacional de Contabilidad. 
Se muestran estas capacidades y elementos, como una introducción al fomento y desarrollo de la cultura profesional del Contador Público en el Perú. Estamos terminando el año 2002, mostrando limitado interés en la trascendencia de cambios en la profesión contable, que se está realizando en las áreas de la gestión de conocimiento $\mathrm{y}$ del capital intelectual, para el presente siglo XXI.

Nuestra apreciación profesional sobre el tema indica que en el Perú todavía se tiene un elevado número de Contadores Públicos que no han tenido la oportunidad de ingresar al estudio de la gestión del conocimiento y del capital intelectual. Guizás esperan que en el transcurso de estos años, se les ofrezcan fórmulas y procedimientos de fácil aplicación y puedan usarlas en las áreas de trabajo contable que actualmente realizan; tal como se presentaron cuando en 1984 se implemento el Plan Contable General Revisado o como en la década del noventa, cuando se plantearon los procesos contables y tributarios de ajustes por inflación.

Pero, consideramos que en este caso no va a ser así. Solamente el Contador Público que se dedique al estudio de estos importantes temas, de manera seria, analítica y profunda, encontrará elementos necesarios para capacitarse y ofrecer sus servicios profesionales con un alto contenido profesional para vislumbrar la gestión del conocimiento en su verdadera aplicación contable y el contenido filosófico del capital intelectual.

En los presentes albores del siglo XXI, nos encontramos ante una nueva época revolucionaria, producida por la explosión de las tecnologías de la información y de las telecomunicaciones, que tienen cada vez más una importancia capital en la configuración de la sociedad de nuestro tiempo actual, hasta tal punto que algunos la llaman "la sociedad de la información", por los signos externos tales como: la telefonía móvil, internet, el comercio electrónico, la banca telefónica, la televisión digital, la televisión por cable, etc. Estas tecnologías de la información y las telecomunicaciones, constituyen medios para transmitir y gestionar contenidos (películas, música, imágenes, acontecimientos en general, etc.) y para gestionar eficazmente datos, información y contenido. Por lo tanto, el conocimiento se convierte en el factor fundamental de creación de riqueza en la sociedad de nuestro tiempo actual, en base a los cuatro factores de creación de riqueza en una economía que son: tierra, trabajo, capital y conocimiento.

Pero la importancia relativa de estos cuatro factores ha ido variando con el tiempo, en las tres etapas en que de una forma simplificada se ha dividido la historia de la humanidad y que en forma cronológica son: la era agraria, la era industrial y la era del conocimiento.

\section{LA EMPRESA COMPETITIVA EN LA SOCIEDAD DEL CONOCIMIENTO}

La empresa competitiva, en el contexto de los mercados globales, es aquella que siguiendo los paradigmas de la teoria moderna del management consigue ventajas competitivas sostenibles. 
El paradigma más significativo que destaca con luz propia es de "Empresa Inteligente" formulada por su autor James Brian Quinn, cuyos cuatro principios que lo fundamentan son:

1. Para conseguir las máximas ventajas competitivas, sostenibles a largo plazo, la "Empresa Inteligente" concentra sus esfuerzos y recursos internos en unas pocas fuentes de conocimientos, servicios o actividades, que crean en la mentalidad de la clientela una imagen de diferenciación y competencia singular.

2. Una vez convencida la "Empresa Inteligente" de cuáles son estos conocimientos, servicios o actividades esenciales y una vez delimitados nitidamente de los demás, se trata de considerar a las actividades como susceptibles de ser realizadas ya sea internamente como externamente. Si se pueden realizar externamente de un modo satisfactorio, se subcontratan.

3. Para mantener una posición triunfadora a lo largo del tiempo, es necesario profundizar en los conocimientos, servicios $y$ actividades básicas y dominarlas cada vez más. Se trata de concentrar todas las energías de la empresa en ser los primeros a nivel mundial, de tal forma que nadie sea capaz de ingresar a esta fortaleza (barreras de entrada), donde se encuentran nuestra fuente de ventajas competitivas.

4. La empresa debe planificar, controlar y vigilar todas las actividades subcontratadas con la finalidad de evitar que puedan producirse situaciones de dependencia o dominio por parte de empresas en las que se ha efectuado la subcontratación.

En este cuerpo teórico de cuatro principios, se pueden establecer jerarquias en cuanto a importancia relativa de las teorias existentes. Así, podemos mencionar las teorias de la estrategia que tienen un carácter relevante, destacando de manera significativa la Teoria de los Recursos y de las Capacidades, cuyo resumen dice: Los recursos y las capacidades de una empresa, juegan un papel cada vez más relevante en la definición de la empresa. La empresa tiene dificultades para plantear qué necesidades quiere satisfacer, por lo que puede preguntarse, alternativamente, a cerca de qué necesidades puede satisfacer. En este último caso, la orientación externa no puede ser el único fundamento de la estrategia empresarial, sino que es preciso recurrir al análisis de los recursos y capacidades disponibles para poner en marcha una estrategia. Se puede afirmar que, cuanto más dinámico sea el entorno de la empresa, más sentido tiene basar su estrategia en los recursos y capacidades de tipo interno, frente a hacerlo sobre consideraciones de tipo externo.

En la Teoria de los Recursos y de las Capacidades, la estrategia tiene que ver con el ajuste de los recursos y capacidades de una empresa a las oportunidades que surgen en su entorno. El punto de partida para la formulación de la estrategia parte de una declaración de la identidad y el propósito de la empresa en su misión y visión; es decir, la empresa debe 
conocerse a sí misma, en el sentido de una profunda y completa comprensión de sus propios recursos y capacidades.

La estrategia basada en los recursos comprende tres elementos claves:

1. Seleccionar-una estrategia que explote los principales recursos y capacidades de la empresa.

2. Asegurar que los recursos de la empresa se empleen completamente y que su beneficio potencial sea explotado al límite.

3. Construir la base de recursos de la empresa.

Para conocer los principales recursos de la empresa, se plantea la siguiente clasificación: Tangibles (Estados contables); Intangibles (Tecnologia y cultura); y en Capital Humano (conocimientos destrezas y capacidades), tal como se presenta esquemáticamente a continuación:

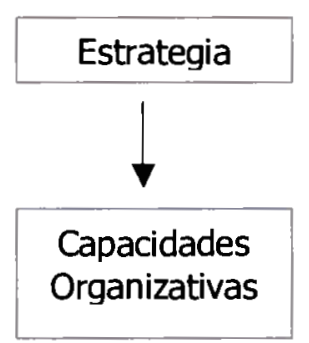

\section{Tangibles}

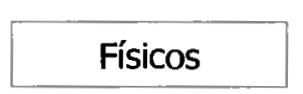

\section{Financieros}

Tecnología

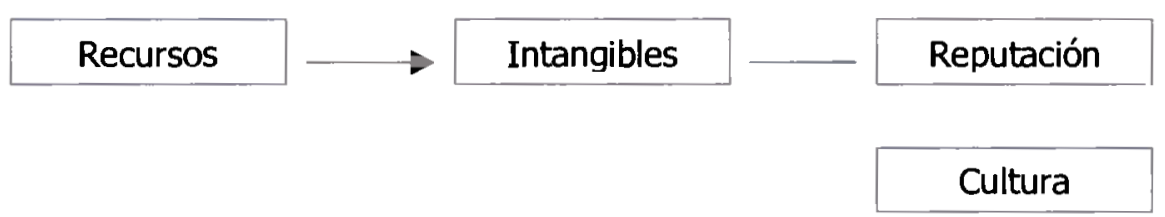

Conocimientos y destrezas

Humanos

Capacidad de comunicación y relación

Motivación 
Este esquema se lo adecúa a los planteamientos del presente artículo como sigue:

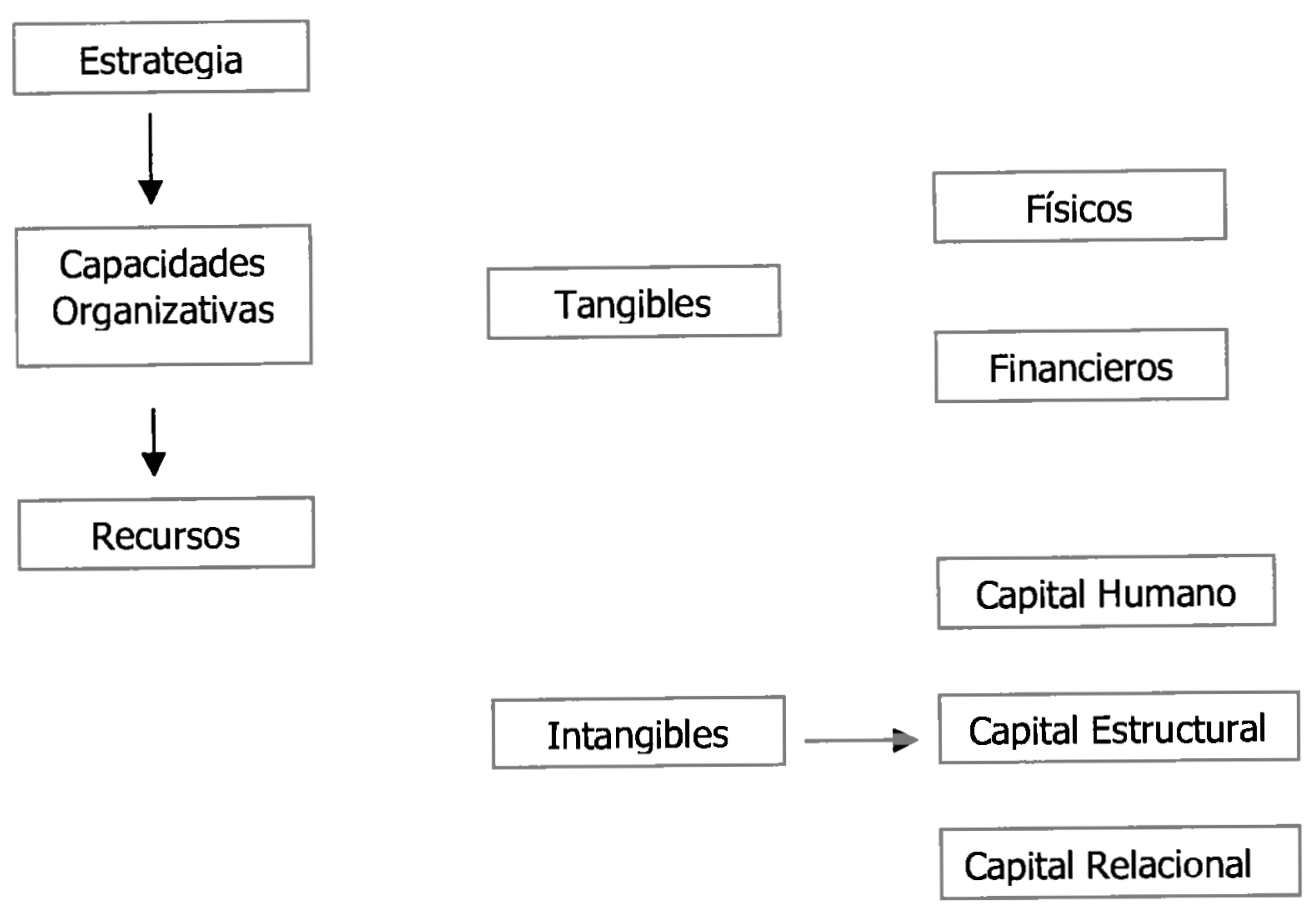

Los recursos normalmente no son productivos por sî mismos. Las tareas productivas requieren la cooperación de grupos de recursos. El término -Capacidades Organizativas", empleado en los esquemas presentados, se refiere a la capacidad de la empresa para acometer una actividad concreta. El interés reside aquí en cuanto a las capacidades de relación con otras empresas, es decir, ¿qué puede hacer la empresa mejor que sus competidores?

Para examinar las capacidades que posee la empresa, es necesario hacer una clasificación de sus actividades. Generalmente, se utilizan dos enfoques: el primero, dando una clasificación funcional, que identifica las capacidades organizativas en relación con cada una de las principales áreas funcionales de la empresa; el segundo, la aplicación de la "cadena de valor", que desagrega a la empresa en una cadena de actividades secuenciales. Al respecto, el C.P. Samuel Alberto Mantilla, escribe en su libro ${ }^{2}$ que "el objetivo del análisis de la cadena de valor es identificar los elementos de los procesos y actividades organizacionales con la creación de valor por parte de la empresa. Los procesos son conjuntos estructurados y medibles de actividades, diseñados para producir un resultado específico para un cliente o mercado particular. Identificar el proceso de creación de valor de la empresa -la manera mediante la cual se crea, integra,

2 Capital Intelectual - Contabilidad del Conocimiento. ECOE Ediciones - Asociación Colombiana de Costos y Contabilidad Directiva. 1999 
transforma y utiliza el conocimiento -requerirá una percepción horizontal de la organización y de las relaciones funcionales que existen dentro de ella".

Ya hemos indicado que los conocimientos en la empresa son hoy en dia la principal fuente de ventajas competitivas sostenibles. Es evidente, sin embargo que no todos los conocimientos que la empresa tiene, serán fuentes de ventajas competitivas sostenibles, sino solamente aquellos que contribuyan decisivamente a la generación de valor económico y que son denominados "conocimientos esenciales" y que se pueden considerar, de una perspectiva económico-financiera como "activos intangibles" y "activos intelectuales o capital intelectual”.

\section{EL CAPITAL INTELECTUAL Y LA IMPORTANCIA DE LA GESTIÓN PROFESIONAL}

Aquí ingresa el criterio y la experiencia profesional del Contador
Público, en la búsqueda de metodologías y modelos que contribuyan a gestionar eficazmente dicho Capital Intelectual.

En el actual mundo competitivo, caracterizado por un progreso vertiginoso en las nuevas tecnologías de la información y las telecomunicaciones, los activos más valiosos de las empresas ya no son los activos tangibles, generalmente mostrados en los rubros de "Inmuebles, maquinaria y equipo" y de "Existencias" del Balance General; sino los activos intangibles que tienen su origen en los conocimientos, habilidades, valores y actitudes de las personas que forman parte del núcleo estable de la empresa. A estos activos intangibles se les denomina Capital Intelectual y comprenden todos aquellos conocimientos tácitos o explícitos que generan valor económico para la empresa.

Los activos tangibles se pueden tocar; los activos intangibles no, pero si se pueden identificar y clasificar adecuadamente, tal como se muestra en el siguiente esquema:

\begin{tabular}{|c|c|c|c|c|}
\hline \multicolumn{5}{|c|}{ ACTIVOS } \\
\hline \multicolumn{2}{|c|}{ TANGIBLES } & \multicolumn{3}{|c|}{ INTANGIBLES } \\
\hline \multicolumn{2}{|c|}{ CONTABLES } & \multicolumn{3}{|c|}{ EXTRA-CONTABLES } \\
\hline SEGÚN P.C.G.R. & SEGÚN P.C.G.R. & $\begin{array}{l}\text { COMPETENCIA } \\
\text { INDIVIDUAL }\end{array}$ & $\begin{array}{l}\text { ESTRUCTURA } \\
\text { INTERNA }\end{array}$ & $\begin{array}{c}\text { ESTRUCTURA } \\
\text { EXTERNA }\end{array}$ \\
\hline $\begin{array}{l}\text { - INMUEBLES, MAGUI- } \\
\text { NARIAS Y EQUIPOS } \\
\text { - EXISTENCIA }\end{array}$ & $\begin{array}{l}\text { - CONCESIONES Y DERECHOS } \\
\text { - } \text { PATENTES Y MARCAS } \\
\text { - GASTOS DE INVESTIGACIÓN } \\
\text { DE DESARROLPLORACIÓN } \\
\text { - GASTOS DE ESTUDIOS Y } \\
\text { PROYECTOS } \\
\text { - GASTOS DE PROMOCIÓN Y } \\
\text { PREOPERATIVOS } \\
\text { - GASTOS DE EMISION DE AC- } \\
\text { CIONES Y OBLIGACIONES }\end{array}$ & $\begin{array}{l}\text { CONOCIMIENTOS, } \\
\text { VALORES Y ACTITU- } \\
\text { DES DE PERSONAS } \\
\text { GUE TRABAJAN EN } \\
\text { LA EMPRESA }\end{array}$ & $\begin{array}{l}\text { - MÉTODOS Y PROCE- } \\
\text { DIMIENTOS DE TRA- } \\
\text { BAJO } \\
\text { - SOFTWARE } \\
\text { - } \text { BASES DE DATOS } \\
\text { - INVESTIGACION Y } \\
\text { DESARROLLO } \\
\text { - SISTEMAS DE DI- } \\
\text { RECCIÓNY GESTIÓN } \\
\text { - CULTURA DE LA } \\
\text { EMPRESA } \\
\text { - PROPIEDADES INTE- } \\
\text { LECTUALES }\end{array}$ & $\begin{array}{l}\text { - CARTERA DE CLIEN- } \\
\text { TES } \\
\text { RELACIONES CON } \\
\text { PROVEEDORES, } \\
\text { BANCOS Y ACCIO- } \\
\text { NISTAS } \\
\text { - ACUERDOS }\end{array}$ \\
\hline
\end{tabular}


Los activos intangibles contables anotados, provienen del Plan Contable General Revisado y son reconocidos como tales en la doctrina contable, por cuanto generan un pasivo. Los activos intangibles extra-contables son autogenerados por la empresa en marcha y la doctrina contable aún no ha establecido los procedimientos adecuados para su registro e información.

Los activos intangibles extracontables de "competencia individual", corresponden a la educación, experiencia, conocimientos, habilidades, valores y actitudes de las personas que trabajan en la empresa. No son propiedad de la empresa. La empresa contrata el uso de estos activos con sus trabajadores, quienes al marcharse a casa se llevan consigo estos activos. A este conjunto de activos se les denomina Capital Humano.

Los activos intangibles extracontables de "estructura interna", corresponden a la estructura organizativa formal e informal, a los métodos y procedimientos de trabajo, al software, a las bases de datos, a la investigación y desarrollo, a los sistemas de dirección y gestión, y a la cultura de la empresa. Estos activos son propiedad de la -mpresa y algunos de ellos pueden orotegerse legalmente.

Los activos intangibles extracontables de "estructura externa", zorresponden a la captura de E-entes, a las relaciones con los oroveedores, bancos y accionistas, z. Ios acuerdos de cooperación y ahanzas estratégicas, tecnológicas, producción y comerciales, a las marcas comerciales y a la imagen de la empresa. Estos activos son propiedad de la empresa y algunos de ellos pueden protegerse legalmente.

Los activos intangibles extracontables, que mostramos en estos tres canales últimos, constituyen el capital intelectual, como la principal fuente de riqueza de la empresa y es totalmente lógico que la dirección de la misma preste una atención especial a la gestión eficaz de dicho capital intelectual.

Por lo expuesto, la capacidad de identificar, auditar, medir, renovar, incrementar y en definitiva gestionar estos activos intelectuales, es un factor determinante en el éxito de la empresa de nuestro tiempo.

\section{CONCLUSIONES}

1. El Contador Público Colegiado, necesita contar con un Programa Especial de Educación Continua, relacionado con la gestión del conocimiento y el capital intelectual, para mantener un nivel óptimo de actualización profesional acorde con la explosión de las tecnologias de la información $\mathrm{y}$ de las telecomunicaciones.

2. El Contador Público Colegiado requiere estudiar los variados campos de la gestión del conocimiento como: la empresa competitiva, las ventajas competitivas sostenibles, las teorías de la estrategia, las teorias de los recursos y los procesos para desarrollar capacidades organizativas, que conduzcan a los planteamientos profesionales del capital intelectual y al uso de esta información 
para el desarrollo de las empresas nacionales en el Perú.

3. El Contador Público, por su formación profesional, es el profesional indicado para precisar los procesos cuantificables de los activos intangibles contables en sus dos posiciones: en sus valores históricos con el cumplimiento de los principios de contabilidad y las normas internacionales de contabilidad y en sus valores extracontables que formen parte del capital intelectual de la empresa. Además, es el profesional indicado para dirigir los estudios relacionados con la determinación y cuantificación de los activos intangibles extra-contables de competencia individual, de estructura interna y de estructura externa, que forman parte del capital intelectual de la empresa.

\section{BIBLIOGRAFÍA}

1. Samuel Alberto Mantilla B., 2000, Capital Intelectual-Contabilidad del Conocimiento. Ecoc. EdicionesAsociación Colombiana de Costos y Contabilidad Directiva.

2. XXIV Conferencia Interamericana de Contabilidad, 2001, Área 1: Investigación Contable-Punta del Este-Uruguay.

3. XXIII Conferencia Interamericana de Contabilidad, 1999, Área 1: Investigación Contable-San Juan-Puerto Rico.

4. Brian Quinn James, 1992, Intelligent Enterprise-Free Press.

5. Brian Quinn James, 1996 , Harvard Business Review, March-April, vol. 74, 1996.

6. Ross Johan, 1996 Capital Intelectual: que se puede medir, se puede gestionar-Harvard Deusto Business Review. 